\title{
The Role of Inflammation in Contributing to Vascular Risk in Subclinical Hyperthyroidism: Randomized Controlled Trial
}

\author{
Shaikh Abdul Kader Kamaldeen Abdul Shakoor ${ }^{a}$, b, Hong Li Jiao ${ }^{a}$, \\ Alvin Wai Kit Tan ${ }^{a}$, Liew Huiling ${ }^{\text {a }}$
}

\begin{abstract}
Background: Subclinical hyperthyroidism ( $\mathrm{SH}$, defined by low or undetectable serum thyroid stimulating hormone and normal thyroid hormones) is associated with increased cardiovascular risk (CVR) such as atrial fibrillation. Few studies also showed an increased risk of vascular disease and mortality in SH. Inflammation has been shown to play a significant role in the pathogenesis of cardiovascular disease. Increased levels of C-reactive protein (CRP), lipoprotein associated phospholipase A2 (Lp-PLA2, an inflammatory marker which plays a critical role in atherosclerosis), neutrophil to lymphocyte ratio (NLR) and monocyte to lymphocyte ratio (MLR) have been reported in conditions with increased cardiovascular risk. We aimed to ascertain whether abnormal CRP, Lp-PLA2, NLR and MLR contribute to an increased CVR in SH.
\end{abstract}

Methods: CRP, Lp-PLA2, NLR and MLR in peripheral blood were measured in $30 \mathrm{SH}$ subjects at baseline and after 6 months of treatment with either carbimazole or placebo in a randomized placebocontrolled design.

Results: There was no significant difference in CRP, Lp-PLA2, NLR and MLR between carbimazole and placebo treated groups at 6 months. There was also no statistical difference in the above parameters if we compared the change or difference between two visits (visit 2 and visit 0 levels) in both groups.

Conclusion: There is no evidence of chronic inflammation in our small cohort of SH subjects. Our finding needs to be confirmed in future studies with larger number of $\mathrm{SH}$ subjects.

Keywords: Subclinical hyperthyroidism; Inflammation; Cardiovascular risk

Manuscript submitted December 29, 2020, accepted February 15, 2021

Published online February 24, 2021

aDepartment of Endocrinology, Tan Tock Seng Hospital, Singapore city, Singapore

${ }^{\mathrm{b}}$ Corresponding Author: Shaikh Abdul Kader Kamaldeen Abdul Shakoor, Department of Endocrinology, Tan Tock Seng Hospital, 11 Jalan Tan Tock Seng, Singapore city 308433, Singapore. Email: shaikh_shakoor@ttsh.com.sg

doi: https://doi.org/10.14740/jem723

\section{Introduction}

Subclinical hyperthyroidism (SH) is characterized by low serum concentration of thyroid stimulating hormone (TSH) in the presence of normal serum thyroid hormones and the absence of obvious symptoms of hyperthyroidism. The reported prevalence of SH is variable (depending on the area, iodine intake and inclusion of exogenous $\mathrm{SH}$ ), more common in women than in men (female/male ratio is 1.5:1) and its incidence increases with advancing age [1]. SH is associated with increased prevalence of atrial fibrillation [2] and few but not all studies have shown association of SH with cardiovascular disease [3] and increased mortality [4].

Chronic inflammation has been shown to play a significant role in the pathogenesis of cardiovascular disease [5]. One of the established inflammatory vascular markers, C-reactive protein (CRP) has been shown as an independent risk factor for cardiovascular events [6]. Lipoprotein associated phospholipase A2 (Lp-PLA2), an enzyme produced by inflammatory cells, has been shown to be an independent predictor for future ischemic events [7].

Elevated neutrophil to lymphocyte ratio (neutrophil count divided by lymphocyte count, NLR) and monocyte to lymphocyte ratio (MLR) have been reported as a marker for chronic inflammation, which seems to predict death and major cardiovascular events in patients with ischemic heart disease $[8,9]$.

We hypothesised that $\mathrm{SH}$ was associated with abnormal levels of CRP, Lp-PLA2, NLR and MLR contributing to atherosclerosis and or increased cardiovascular risk. The primary aim of this study was to evaluate the effect of anti-thyroid drug carbimazole (compared to placebo) on the inflammatory vascular markers such as CRP, Lp-PLA2, NLR, and MLR in peripheral blood of SH subjects. Our secondary aim was to evaluate anthropometric measurements in $\mathrm{SH}$ after treatment with carbimazole compared to placebo.

\section{Materials and Methods}

Thirty SH patients, aged 21 to 70 years old were recruited from Endocrine Clinic at Tan Tock Seng Hospital, a tertiary hospital in Singapore after the diagnosis has been confirmed at least on two occasions 6 to 8 weeks apart, showing low serum TSH with 
normal thyroid hormones, both free thyroxine (FT4) and free triiodothyronine (FT3). All study subjects had milder form of $\mathrm{SH}$ with detectable serum TSH due to multinodular goiter. Exclusion criteria included sick euthyroid state, recent radioiodine therapy (within 1 year of screening visit), pregnant or breastfeeding patients, and acute medical illnesses such as infections and active cancer. Due to difficulty in recruitment, we included patients with cardiovascular risk factors: $\%$ of patients in the placebo vs carbimazole group, diabetes mellitus: 33\% versus $42 \%$, hypertension: $44 \%$ versus $42 \%$, hyperlipidemia: $33 \%$ versus $33 \%$ and ischemic heart disease: $6 \%$ versus $8 \%$.

At the initial visit (visit 0), all subjects had anthropometric measurements (height, weight, body mass index (BMI), blood pressure, fasting bloods for CRP, Lp-PLA2, NLR and MLR). SH patients were then randomized electronically in blocks of 4 using randomization codes by a statistician (for carbimazole or placebo) to receive either carbimazole or placebo in a parallel group trial design. The study investigators and the study subjects were blinded in the randomization process.

At 12 weeks (visit 1), thyroid function test (TFT) was repeated and if necessary, dose of carbimazole was amended to achieve biochemical euthyroid state (Fig. 1). Similar adjustment in the dosage (number of tablets) was done for those on placebo tablets. This dosage adjustment was done by the research coordinator, who was not blinded, in discussion with the principal investigator who was blinded in the process. All subjects had similar measurements at the final visit at 6 months (18 in placebo group and 12 in carbimazole group completed the study). The recruitment period was from November 2012 to January 2018.

\section{Biochemical measurements}

Serum TSH, FT4 and FT3 were performed on two Beckman Coulter DxI-800 immunoassay analyzers using manufacturersupplied reagents and calibrators. Locally derived $95 \%$ reference intervals for each of the assays were: FT4 8 - $21 \mathrm{pmol} / \mathrm{L}$, FT3 3.5 - 6.0 pmol/L, TSH 0.34 - $5.6 \mathrm{mU} / \mathrm{L}$. Serum CRP was performed after collecting venous blood in lithium heparin containing vacutainer by rate turbidometry method with normal reference value between 0 and $5 \mathrm{mg} / \mathrm{L}$.

\section{Lp-PLA2}

Serum Lp-PLA2 levels were measured by enzyme linked immunosorbent assay (ELISA) method using a commercially available kit from RayBiotech (RayBio Human Lp-PLA2 Elisa kit, Georgia, United States). The serum was in a dilution of $2 \mathrm{x}$ for the assay, following the manufacturer's instructions. The intra-assay coefficient variation was below 36\%. All samples were analyzed at the end of the study from the frozen serum samples which were stored in the freezer at -20 to $80^{\circ} \mathrm{C}$.

\section{NLR and MLR}

This was performed after collecting venous blood in ethylene

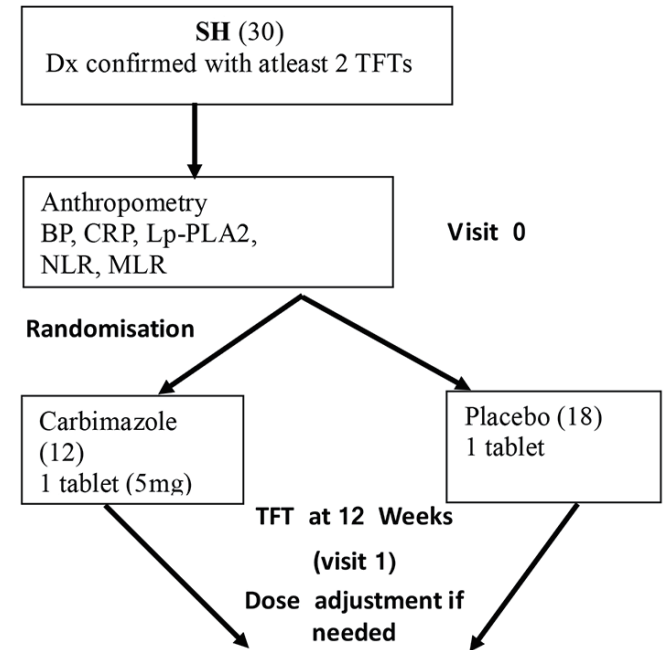

TSH $<0.1 \mathrm{mU} / 1$ and FT4 8-21 pmol/1: Increase CBZ/PLB 2 tab/d

TSH 0.1-0.34 mU/1 and FT4 8-21 pmol/1: Increase CBZ/PLB $1.5 \mathrm{tab} / \mathrm{d}$

TSH 0.34-4 mU/1 and FT4 8-21 pmol/: maintain CBZ/PLB $1 \mathrm{tab} / \mathrm{d}$

TSH 4.1-12 mU/l and FT4 $<8 \mathrm{pmol} / \mathrm{l}$ : Decrease CBZ/PLB half tab/d

PS: If FT $4>21 \mathrm{pmol} / 1$ or TSH $>12 \mathrm{mU} / 1$, to withdraw from study

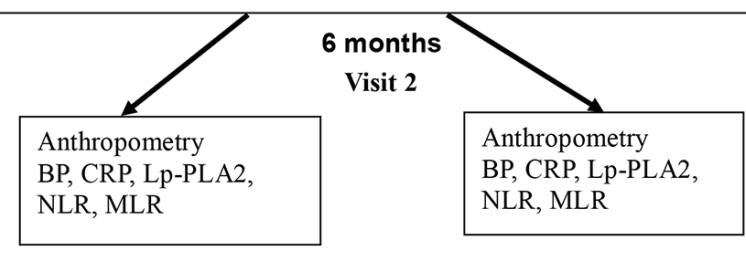

End of study

Figure 1. SH study flow chart. SH: subclinical hyperthyroidism; BP: blood pressure; CRP: C-reactive protein; Lp-PLA2: lipoprotein associated phospholipase A2; NLR: neutrophil to lymphocyte ratio; MLR: monocyte to lymphocyte ratio; TSH: thyroid stimulating hormone; FT4: free thyroxine; PLB: placebo.

diamine tetraacetic acid (EDTA) containing vacutainer for full blood count (FBC). Impedance counting/flow cytometry method was used. The reference values for neutrophil, monocyte, and lymphocyte counts are $1.9-6.6 \times 10^{9} / \mathrm{L}(40-74 \%)$, $0.2-0.7 \times 10^{9} / \mathrm{L}(4-10 \%)$, and $1.1-3.1 \times 10^{9} / \mathrm{L}(17-49 \%)$ respectively.

\section{Statistical analysis}

This was done using Software for Statistics and Data Science (STATA) 13. There was no previous study on Lp-PLA2, NLR, MLR and effect of treatment on the above markers in SH patients to calculate the sample size. We wished to recruit 40 $\mathrm{SH}$ patients in total but could not achieve this target due to difficult recruitment. The comparison between carbimazole and placebo was assessed using Student's $t$-test for normally distributed data (described using mean and standard deviation, SD). The Wilcoxon rank sum test was used to assess for comparisons for the data which were not normally distributed, and their results were described using median and range. The results were declared as being significant if P-value is less 
Table 1. Comparisons Between SH Patients on Carbimazole and Placebo at Baseline and at 6 Months

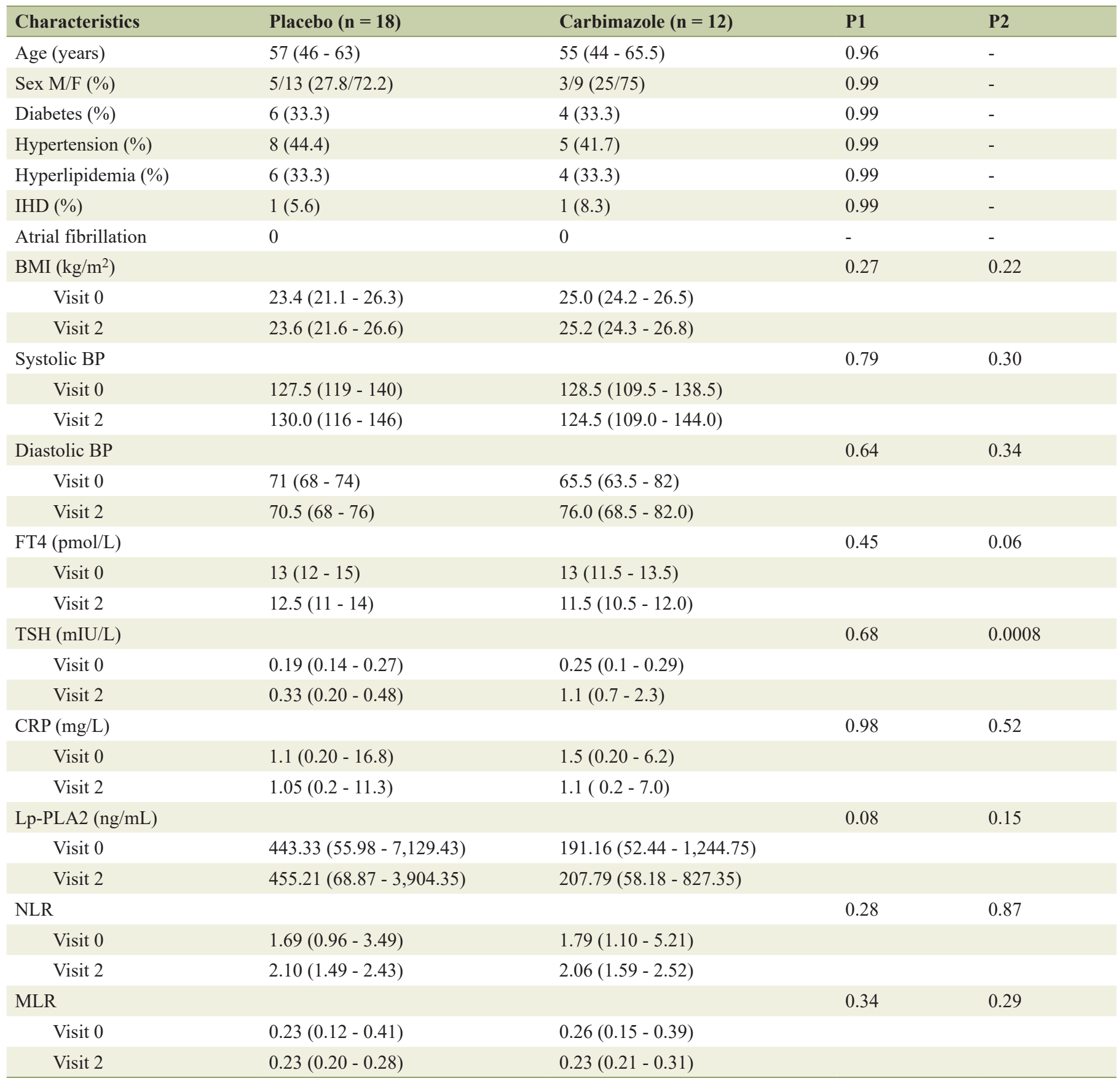

All data except age and sex are expressed as median and range. $\mathrm{P} 1$ and $\mathrm{P} 2$ are the differences between placebo and carbimazole groups at visit 0 and visit 2, respectively. IHD: ischemic heart disease; BMI: body mass index; BP: blood pressure; TSH: thyroid stimulating hormone; FT4: free thyroxine; CRP: C-reactive protein; Lp-PLA2: lipoprotein associated phospholipase A2; NLR: neutrophil to lymphocyte ratio; MLR: monocyte to lymphocyte ratio.

than 0.05 .

\section{Results}

There was no significant difference between the two groups at baseline in relation to age, sex, BMI, blood pressure, TSH, FT4, and the presence of vascular markers such as hematocrit (HT), hyperlipidemia, diabetes, atrial fibrillation and ischemic heart disease.

Serum TSH improved in the carbimazole treated group at 6 months (median of 1.1 versus $0.33 \mathrm{mU} / \mathrm{L}, \mathrm{P}=0.0008$ ). Se- 
rum FT4 also improved after treatment with carbimazole but did not reach statistically significance (from a median of 13 to $11.5 \mathrm{pmol} / \mathrm{L})$.

We did not find any significant difference in the levels of CRP, Lp-PLA2, NLR and MLR between carbimazole and placebo groups (Table 1). There was also no statistical difference in the above parameters if we compared the change or difference between two visits (visit 2 and visit 0 levels) in both groups.

\section{Discussion}

We report for the first time that inflammatory markers, including well known marker (CRP) and the newer markers such as Lp-PLA2, NLR and MLR are not affected in our small cohort of SH subjects. The above mentioned inflammatory markers did not change significantly in the carbimazole treated group compared to the placebo group at 6 months despite the former group achieving euthyroid state with normalization of serum TSH.

Few studies have evaluated serum CRP levels in hyperthyroidism. Only one study reported higher CRP in 250 hyperthyroid subjects compared to controls but the same study reported much higher CRP levels in hypothyroid subjects [10]. Two studies evaluated CRP levels in SH subjects and both reported no change in CRP levels compared to euthyroid group $[11,12]$. Bilgir et al also evaluated CRP levels 3 months after treatment with propyl thiouracil and reported no significant change in CRP levels after using anti-thyroid drug [11]. In our study, we also found no significant change in CRP levels after carbimazole suggesting absence of inflammatory state in $\mathrm{SH}$.

There are no previous studies on Lp-PLA2 in SH or overt hyperthyroidism. Even though this novel inflammatory marker has been shown to be an independent vascular marker, we did not find any difference in their levels after carbimazole.

Similarly, there are no previous studies evaluating NLR and MLR in SH. One study studied NLR in subjects with hyperthyroidism due to Graves' disease and reported low NLR in Graves' disease subjects compared to controls [13]. However, in our study, all SH subjects had nodular goiter rather than due to Graves' disease and even in this group, we did not find any significant change in NLR or MLR. Hence, these results again suggest lack of chronic inflammatory state in $\mathrm{SH}$.

The strengths of this study include evaluation of four different inflammatory markers in SH subjects using randomized placebo-controlled model. The disadvantage of this study includes smaller number of SH subjects mainly due to difficulty in recruitment and studying few $\mathrm{SH}$ subjects with established cardiovascular risks.

From the clinical perspective, current guidelines advocate treatment for older subjects with grade $2 \mathrm{SH}(\mathrm{TSH}<0.1$ $\mathrm{mU} / \mathrm{L}$ ) due to associated morbidities such as atrial fibrillation and selected symptomatic younger SH subjects based on observational studies $[14,15]$. As of now, randomized controlled trials (RCTs) showing cardiovascular benefit with treatment of SH is lacking. Hence, we studied whether treating SH improves the inflammatory markers. However, we could not prove existence of chronic inflammation in small number of SH subjects in our study.

\section{Conclusion}

There is no evidence of chronic inflammation in our small cohort of SH subjects. Our finding needs to be confirmed in future studies with larger number of SH subjects.

\section{Acknowledgments}

We thank all clinicians in the Department of Endocrinology, Tan Tock Seng Hospital who have provided their patients and help in the recruitment of study participants.

\section{Financial Disclosure}

National Healthcare Group-Small Innovative Grant, SIG/12011.

\section{Conflict of Interest}

None to declare.

\section{Informed Consent}

All subjects gave their written informed consent and local ethics committee approved the study (National Health Group Domain Specific Review Board; NHG DSRB Ref: 2011/02144). The protocol was registered retrospectively on March 4, 2020 in ISRCTN registry with study ID ISRCTN13184358.

\section{Author Contributions}

SAKKAS was the principal investigator and the recipient of the NHG-KTPH grant for this study, involved in all aspects of the study including obtaining local ethics approval, recruitment, conducting, data collection, analyzing the data and writing up the manuscript. HLJ was the research nurse involved in recruiting, conduct of the study and data entry. AWKT and LHL were involved in recruitment, contributing their own patients for the study, analyzing the results and writing up manuscript. All authors have read and approved the manuscript.

\section{Data Availability}

The data that support the findings of this study and the protocol are available on request from the corresponding author. 


\section{References}

1. Hollowell JG, Staehling NW, Flanders WD, Hannon WH, Gunter EW, Spencer CA, Braverman LE. Serum TSH, $\mathrm{T}(4)$, and thyroid antibodies in the United States population (1988 to 1994): National Health and Nutrition Examination Survey (NHANES III). J Clin Endocrinol Metab. 2002;87(2):489-499.

2. Cappola AR, Fried LP, Arnold AM, Danese MD, Kuller LH, Burke GL, Tracy RP, et al. Thyroid status, cardiovascular risk, and mortality in older adults. JAMA. 2006;295(9):1033-1041.

3. Vadiveloo T, Donnan PT, Cochrane L, Leese GP. The Thyroid Epidemiology, Audit, and Research Study (TEARS): morbidity in patients with endogenous subclinical hyperthyroidism. J Clin Endocrinol Metab. 2011;96(5):13441351.

4. Gussekloo J, van Exel E, de Craen AJ, Meinders AE, Frolich M, Westendorp RG. Thyroid status, disability and cognitive function, and survival in old age. JAMA. 2004;292(21):2591-2599.

5. Libby P, Ridker PM, Maseri A. Inflammation and atherosclerosis. Circulation. 2002;105(9):1135-1143.

6. Ridker PM, MacFadyen J, Libby P, Glynn RJ. Relation of baseline high-sensitivity C-reactive protein level to cardiovascular outcomes with rosuvastatin in the Justification for Use of statins in Prevention: an Intervention Trial Evaluating Rosuvastatin (JUPITER). Am J Cardiol. 2010;106(2):204-209.

7. Oei HH, van der Meer IM, Hofman A, Koudstaal PJ, Stijnen T, Breteler MM, Witteman JC. Lipoprotein-associated phospholipase A2 activity is associated with risk of coronary heart disease and ischemic stroke: the Rotterdam Study. Circulation. 2005;111(5):570-575.

8. Dentali F, Nigro O, Squizzato A, Gianni M, Zuretti F, Grandi AM, Guasti L. Impact of neutrophils to lympho- cytes ratio on major clinical outcomes in patients with acute coronary syndromes: A systematic review and meta-analysis of the literature. Int J Cardiol. 2018;266:3137.

9. Fan Z, Li Y, Ji H, Jian X. Prognostic utility of the combination of monocyte-to-lymphocyte ratio and neutrophilto-lymphocyte ratio in patients with NSTEMI after primary percutaneous coronary intervention: a retrospective cohort study. BMJ Open. 2018;8(10):e023459.

10. Czarnywojtek A, Owecki M, Zgorzalewicz-Stachowiak M, Wolinski K, Szczepanek-Parulska E, Budny B, Florek $\mathrm{E}$, et al. The role of serum $\mathrm{C}$-reactive protein measured by high-sensitive method in thyroid disease. Arch Immunol Ther Exp (Warsz). 2014;62(6):501-509.

11. Bilgir O, Bilgir F, Topcuoglu T, Calan M, Calan O. Comparison of high-sensitivity C-reactive protein and fetuinA levels before and after treatment for subjects with subclinical hyperthyroidism. Endocrine. 2014;45(2):244248.

12. Lee WY, Suh JY, Rhee EJ, Park JS, Sung KC, Kim SW. Plasma CRP, apolipoprotein A-1, apolipoprotein B and Lpa levels according to thyroid function status. Arch Med Res. 2004;35(6):540-545.

13. Turan E. Evaluation of neutrophil-to-lymphocyte ratio and hematologic parameters in patients with Graves' disease. Bratisl Lek Listy. 2019;120(6):476-480.

14. Ross DS, Burch HB, Cooper DS, Greenlee MC, Laurberg P, Maia AL, Rivkees SA, et al. 2016 American Thyroid Association guidelines for diagnosis and management of hyperthyroidism and other causes of thyrotoxicosis. Thyroid. 2016;26(10):1343-1421.

15. Biondi B, Bartalena L, Cooper DS, Hegedus L, Laurberg P, Kahaly GJ. The 2015 European Thyroid Association guidelines on diagnosis and treatment of endogenous subclinical hyperthyroidism. Eur Thyroid J. 2015;4(3):149163. 